\title{
117. Nucleoli in the Morula and the Blastula of Triturus pyrrhogaster
}

\author{
By Osamu Nakamura and Tetsuo Isoya \\ Osaka Kyôiku University, Osaka \\ (Comm. by Yô K. OKadA, M. J. A., June 12, 1968)
}

It has been repeatedly described (Colombo '49, Elsdale et al. '58, Karasaki '59, Wallace '63, Brown and Gurdon '64) and generally accepted (Ebert '65, Waddington '66, Okada T. S. '66) that the nucleolus is lacking in cleaving embryos of amphibians and makes its appearance at the beginning of gastrulation. Further contributions have been added by Karasaki ('64, '65), reporting that a primary component of the nucleolus consisting of only fibrous materials first appears at the late blastula stage and, later on, accumulate ribosome-like particles around it during gastrulation.

The present authors, however, detected a body referable to the primary nucleolus in urodele embryos at stages as early as the morula and the early blastula. The fine structure of this body will be described below.

Materials and methods. As the material were employed embryos of Triturus pyrrhogaster exclusively. They were varied in developmental phases from st. 7 ( 64 cell stage) to st. 9 (early blastula) of Okada and Ichikawa's standard table. After an hour fixation at $0-4{ }^{\circ} \mathrm{C}$ of the whole embryo in phosphate-buffered $5 \%$ glutaraldehyde ( $\mathrm{pH}$ 7.3) containing $0.22 \mathrm{M}$ sucrose, a small piece was excised from each of the animal pole and the dorsal marginal zone. After washed with phosphate buffer, the piece was postfixed for 30 to 60 minutes with $1 \%$ osmium tetroxide buffered at $\mathrm{pH}$ 7.3. Then, the routine dehydration and embedding were carried out with ethanol and epoxy resin (Epon 812) respectively. Sections were cut with LKB ultramicrotome, first, relatively thick (more than 2,000 A) and examined under the phase microscope. Being thus detected the nucleus in the blastomere of large size, the nuclear region alone was cut into serial sections as thin as 500-800 A. Uranyl acetate was used for staining with or without counterstaining by the method of Reynolds ('63). The observation was performed with JEM-7A type electron microscope.

Observations. In the interphase nucleus of both animal pole and marginal zone of the early blastula at st.9, was discovered a small nucleolus as shown in Fig. 1a. It is roughly spherical in shape 
and measures about $1.0 \mu$ in diameter.

When observed with low power magnification, it has an extremely compact and glanular appearance (Fig. 1b). However, higher magnification reveals that it is chiefly composed of a fibrous element,

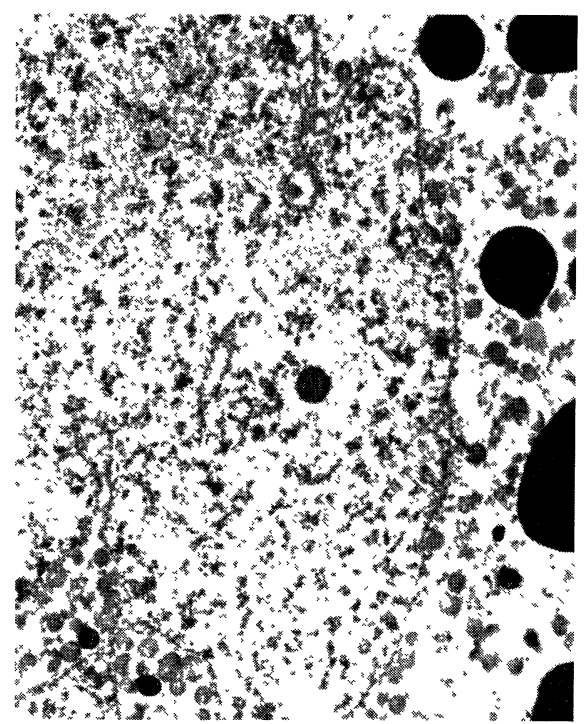

a
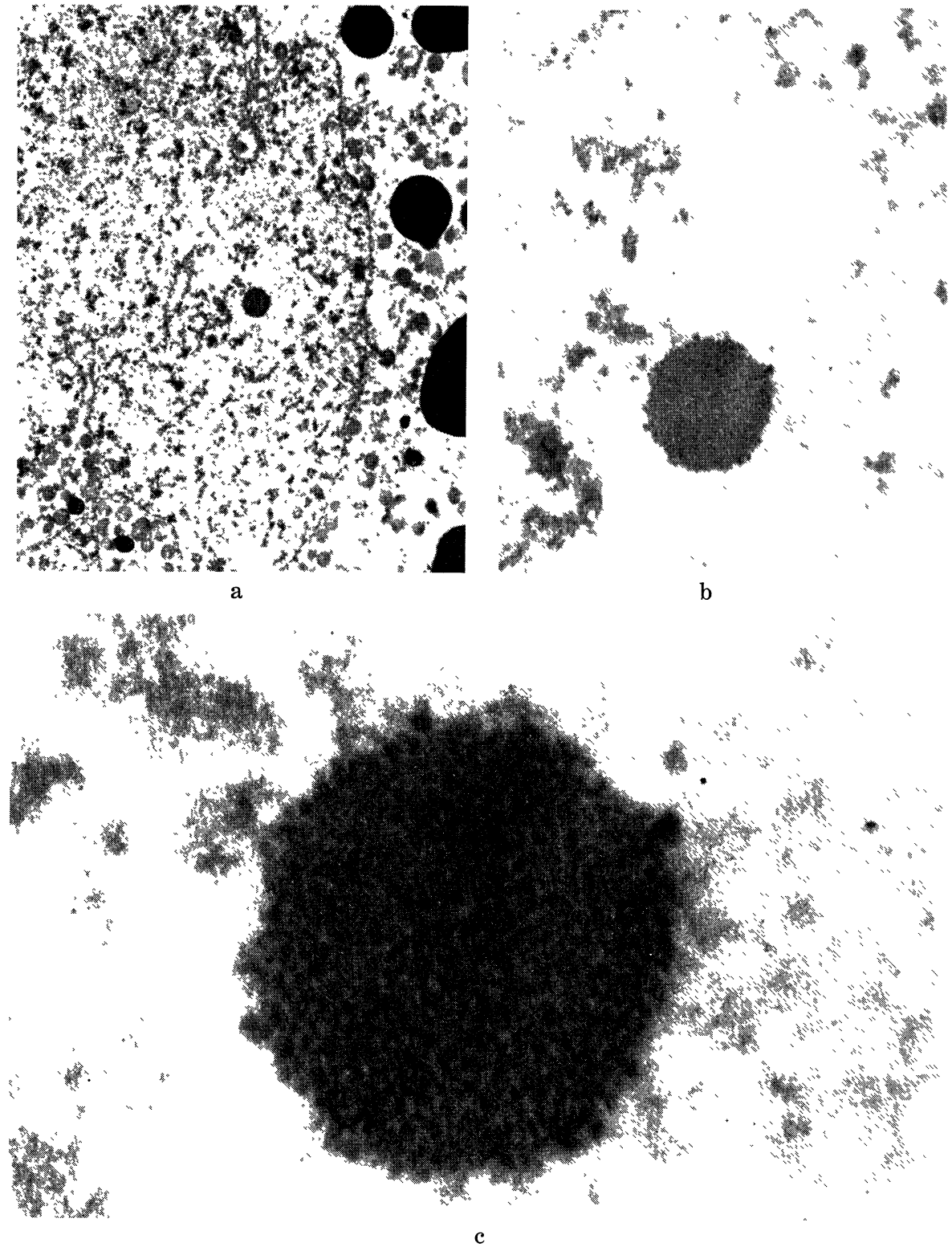

Fig. 1. Primary nucleolus of the early blastula. (st. 9).

a. A part of nucleus containing a nucleolus. $\times 5,000$.

b. Enlargement of the nucleolus. $\times 20,000$.

c. High magnification of the same. $\times 61,000$. 
the diameter of which is 40 to $100 \mathrm{~A}$ (Fig. 1c). This feature closely resembles the central component of the typical nucleolus in the cells of adult animals and probably identical with the "primary nucleolus"

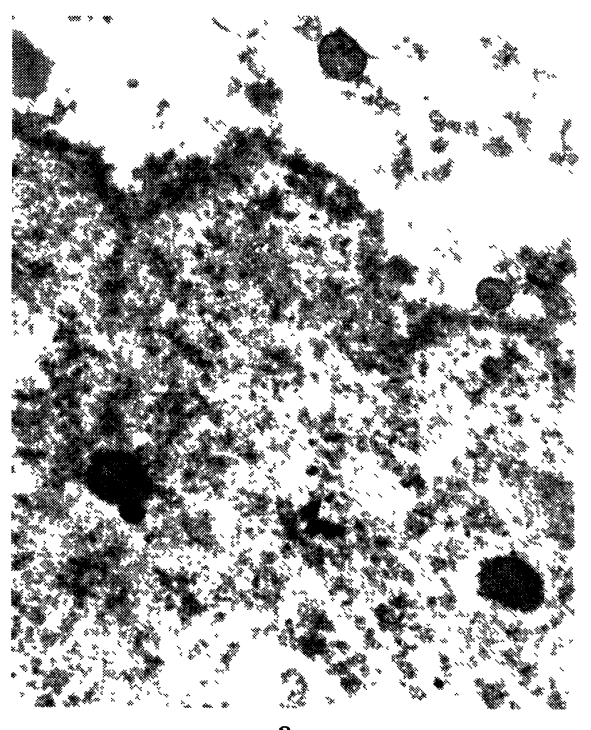

a

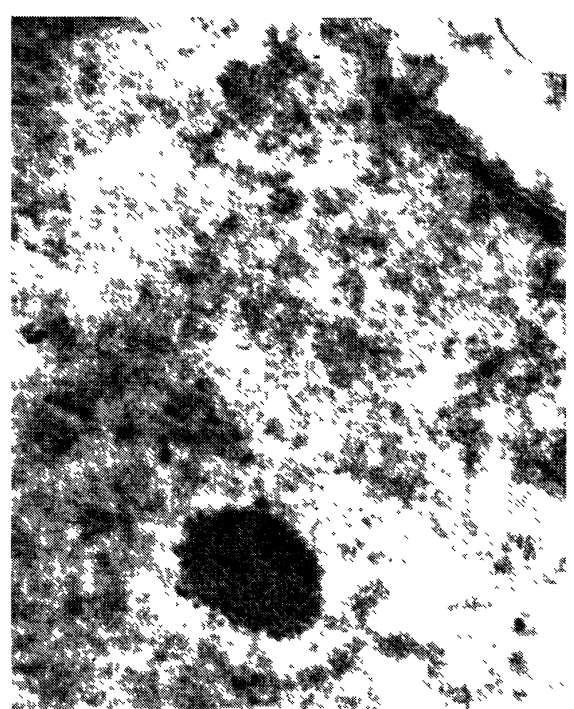

b

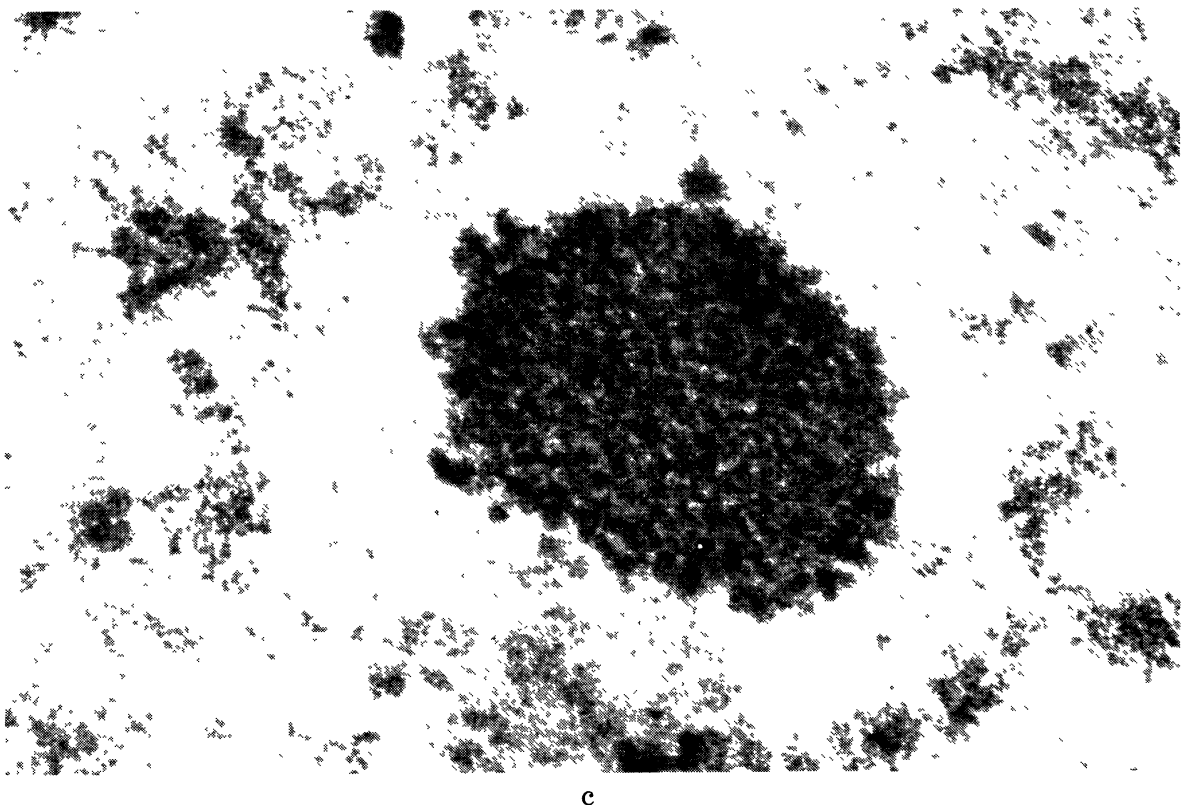

Fig. 2. Primary nucleoli of the early morula (intermediate stage between 7 and 8).

a: A part of nucleus containing two nucleoli. $\times 8,800$.

b: Enlargement of the right one of the nucleoli. $\times 22,000$.

c: High magnification of the same. $\times 69,000$. 
found by Karasaki ('64, '65) in the embryo at st. 10. It is furnished with a small number of ribosome-like particles, 150 to $300 \mathrm{~A}$ in diameter, usually in the central region and rarely on the periphery.

At st. 8, a similar structure referable to the primary nucleolus was found in almost every cell in both animal pole and marginal zone. It is somewhat smaller than that of st. 9, its diameter measuring around $0.6 \mu$.

Even in the earliest phase of morula (intermediate stage between 7 and 8 ), the same bodies were detected though very small in size, ranging from 0.2-0.4 in diameter (Fig. 2a). Few ribosome-like particles $(150-300 \mathrm{~A})$ were rarely recognized within the fibrous structure of the body.

The chromatin material is sparsely and unevenly distributed throughout the interphase nucleus at stages 7-9. As shown in Figs. 1b and $2 \mathrm{~b}$, clumps of chromatin fibrils are found to be connected with the nucleolus. Interchromatin granules are also present as early as at st. 7-8. They are larger (300-500 A) and more opaque than ribosome-like particles, and have rather irregular shape.

Discussion. The high power electron micrograph (Figs. 1c and 2c) revealed that the fine structure of the body in question is very similar to that of the "primary nucleolus" of Karasaki ('64, '65). The latter has been believed so far to be formed at st. 10. In the present study, however, the same body was found even in the earliest phase of morula (intermediate stage between 7 and 8). The reason why it has not been observed until now in embryos earlier than st. 10 may be attributed to its smaller size and fewer number; the smallest measures only $0.2 \mu$ in diameter. At any rate two nucleoli are contained in each nucleus (Fig. 2a) which is in turn present one to each blastomere of large size at earlier morula stages. The present authors were just able to detect them after careful examination of the serial sections of each nucleus. They grow gradually to attain a diameter of $0.6 \mu$ at st. 8 and $1.0 \mu$ at st. 9 .

It is noteworthy that the time of their appearance coincides with the establishing period of the differentiation capacity of marginal zone as demonstrated by Nakamura and Matsuzawa ('67). However, the same structure was found not only in the marginal zone, but also in the animal pole region at about the same time. It may be supposed as one of the possibilities that the primary nucleoli in these two areas differ in their chemical constitution in spite of close resemblance in their fine structure. Another possibility is that additional factors distributed differentially may be involved in the process of bringing about the difference in potency between these two areas. It is not unlikely that such factors interact with the 
primary nucleoli which are quite similar in both regions.

Karasaki ('64, '65) reported that ribosome-like particles appear first within the primary nucleolus during gastrulation, and later accumulate in the peripheral region. In accordance with his opinion, the granulous component was lacking on the periphery of the primary nucleolus newly discovered in cleaving embryos earlier than st. 10 . On the other hand, a few of ribosome-like particles, 150-300 A in diameter, were detected within the fibrous nucleolus even at the earliest stage observed in the present study.

Marinozzi ('62), Swift ('62), Birnstiel et al. ('63), and Jones ('65) emphasized the similarity between nucleolar particles and cytoplasmic ribosomes. According to Chipchase and Birnstiel ('63), base sequences of nucleolar RNA are identical with those of ribosomal RNA. Karasaki ('64, '65) also demonstrated uridine incorporation into RNA in the primary nucleolus of the early gastrula, suggesting that the fibrils in the primary nucleolus participate in the synthesis of ribosomal RNA. From this point of view, the existence of the ribosome-like particles within the primary nucleolus of the morula may be interpreted as an evidence that the synthesis of ribosomal RNA makes its start before cleavage comes to its end.

On the contrary, Gurdon and Brown ('65) proved the suppression of ribosomal RNA synthesis by the cytoplasm of cleaving embryos. Further, Shiokawa and Yamana ('67) extracted an inhibitor of ribosomal RNA synthesis from dissociated cells of the blastula of Xenopus. These evidences are not inconsistent with the idea that the synthesis of the same substance has its start during the cleaving period. A possible explanation may be that, at the beginning of morula stage, concentration of the inhibiting substance decreases to come just below the threshold value required for the complete suppression of the synthesis.

Anyway, the synthesis of ribosomal RNA during cleavage and gastrulation is not an absolute necessity for the progress of early embryonic differentiation, because it was evidenced by Wallace ('60) and Brown and Gurdon ('64) that anucleolate embryos of Xenopus were capable of developing normally up to hatching. Even in these embryos, however, densely fibrous masses identical with the primary nucleolus were observed by Jones ('65). It seems probable that the primary nucleolus contributes to the differentiation of the presumptive regions of ectoderm and mesoderm by forming a basis of functions other than ribosomal RNA synthesis. Further studies on the role played by the primary nucleolus in the differentiation are now in progress in our laboratory. 


\section{References}

Birnstiel, M. L., Fleissner, E., and Hyde, B. B. (1963): Biochim. Biophys. Acta, 76, 454 .

Brown, D. D., and Gurdon, J. B. (1964): Proc. Natl. Acad. Sci. U. S., 51, 139.

Chipchase, M. I. H., and Birnstiel, M. L. (1963): Proc. Natl. Acad. Sci. U. S., 50, 1101.

Colombo, G. (1949): Rend. Accad. naz. Lincei, Ser. VIII, 6, 370.

Ebert, J. D. (1965): Interacting Systems in Development. Holt, Rinehart and Winston, New York.

Elsdale, T. R., Fischberg, M., and Smith, S. (1958): Exp. Cell Res., 14, 642.

Gurdon, J. B., and Brown, D. D. (1965): J. Mol. Biol., 12, 27.

Jones, K. W. (1965): J. Ultrastr. Res., 13, 257.

Karasaki, S. (1959): Embryologia, 4, 273.

- (1964): J. Cell Biol., 23, 48A. (1965): J. Cell Biol., 26, 937.

Morinozzi, V. (1962): Proc. 5th. Intern. Conf. EM. Philadelphia, 2.

Nakamura, O., and Matsuzawa, T. (1967): Embryologia, 9, 223.

Okada, T. S. (1966): Development and Differentiation (in Japanese). Iwanami, Tokyo.

Okada, Yô K., and Ichikawa, M. (1947): Ann. Rep. Exp. Morph., 3, 1.

Reynolds, E. S. (1963): J. Cell Biol., 17, 208.

Swift, H., 1962., in Allen, J, M. (Ed.): Molecular Control of Cellular Activity, 106. McGraw-Hill, New York.

Waddington, C. H. (1966): Principles of Development and Differentiation. Macmillan, New York.

Wallace, H. (1963): J. Morph., 112, 261. 